\title{
Changes in Eyebrow Position and Movement with Aging
}

\author{
Jeongseob Park ${ }^{1}$, Sangho Yun ${ }^{2}$, Daegu Son ${ }^{1}$ \\ ${ }^{1}$ Department of Plastic and Reconstructive Surgery, Keimyung University School of Medicine, Daegu; ${ }^{2}$ Daniel Plastic Surgery, Changwon, \\ Korea
}

Background This study evaluated dynamic changes in eyebrow position related to aging.

Methods Female participants were recruited and separated into two groups aged 20-30 years (the younger group, $\mathrm{n}=20$; mean age, 24.8 years) and $50-70$ years (the older group, $n=20$; mean age, 55.8 years). Photogrammetry was used to determine the eyebrow position at the medial canthus (MC), lateral limbus, lateral canthus, and lateral end point (EP) for 6 actions: smooth opening (the reference action) and closing of the eye, forward gaze, maximum opening and closing of the eye, and maximum frown. Videos were also recorded.

Results No differences in eyebrow position were detected at the MC when opening or closing the eyes smoothly, gazing straight ahead, or closing the eyes maximally. For all 6 actions, the position of the lateral EP in the older group was significantly lower than in the younger group $(P=0.003)$, and the smallest degree of vertical movement at this point was found in both age groups $(\mathrm{P}<0.001)$. Vertical movement at the 4 landmarks of the eyebrows decreased with aging.

Conclusions Eyebrow position was unchanged at the MC with aging, except at maximal eye opening and maximal frown. No differences in eyebrow position were observed between the younger and older groups when eyes were maximally closed, except at the EP. It is important to focus on correction of the lateral EP for periorbital rejuvenation.

Keywords Aging / Physiology / Anthropometry / Eyebrow / Female
Correspondence: Daegu Son Department of Plastic and Reconstructive Surgery, Keimyung University School of Medicine, 56 Dalseong-ro, Jung-gu, Daegu 41931, Korea

Tel: +82-53-250-7636

Fax: +82-53-255-0632

E-mail: handson@dsmc.or.kr

This article was presented at the 6 th Research and Reconstructive Forum of the Korean Society of Plastic and Reconstructive Surgeons on April 7-8, 2016 in Seoul, Korea.

No potential conflict of interest relevant to this article was reported.

\section{INTRODUCTION}

Facial aging is particularly noticeable around the orbit. Drooped eyebrows may create a tired or depressed appearance [1]. However, blepharoplasty without eyebrow lift surgery may result in disharmony between the eye and eyebrow, leading to an aggravated facial appearance.

Many authors have examined the ideal eyebrow position and shape to obtain an aesthetically satisfying surgical result. In 1974, Westmore [2] described the characteristics of the ideal eyebrow as an arch where the brow apex terminates above the lateral limbus of the iris, and the medial and lateral ends of the brow are at the same horizontal level. Ellenbogen [3] and Brennan [4] expressed support for this model. Although research has sought to categorize various ideal eyebrow characteristics according to gender [5], intercanthal distance [6], facial features [7], culture, and age [8], a standardized ideal eyebrow does not exist. Therefore, it is important to know the ways in which the position and shape of eyebrows change with age and what may cause these changes. 
Eyebrows are held in a neutral position by a dynamic balance between soft tissues, such as ligaments and nerves, and the muscles beneath the dermis of the eyebrows. These muscles are the frontalis muscle, orbicularis oculi muscle (OOM), and corrugator supercilii muscle (CSM). The action of these soft tissues and muscles cause the eyebrows to move [9]. If the dynamic balance between these anatomical features is altered, the appearance of eyebrows can become asymmetric and they may droop under the influence of gravity $[10,11]$. It remains unclear how the reciprocal action of the muscles functions and how the eyebrows change with age.

The purpose of this study was to understand the effects of aging on eyebrows by comparing measurements of the position and movement of the eyebrow in younger and older age groups, and to determine which parts of the eyebrow show the greatest change with aging.

\section{METHODS}

Approval for this study was obtained from the Institutional Review Board of Keimyung University Dongsan Medical Center (application no. 10-81). For this study, female participants were recruited and separated into two groups aged 20-30 years (the younger group, $n=20$; mean age, 24.8 years) and $50-70$ years (the older group, $\mathrm{n}=20$; mean age, 55.8 years). Participants were excluded if they had previously undergone periorbital or forehead surgery, had experienced plucking of the eyebrow margin, had suffered from any ophthalmologic or neurologic disease, or had used botulinum toxin. All experiments were performed in the same place and under the same conditions. All subjects were seated in a relaxed position and gazed forward, facing the examiner. Four landmarks were positioned on the face, with a $10-\mathrm{mm}$ ruler for reference. These were the points where the upper margin of the eyebrows met with vertical lines drawn from the medial canthus (A, a), the lateral limbus $(B, b)$, the lateral canthus $(\mathrm{C}, \mathrm{C})$, and the lateral end point of the eyebrows (D, d) (Fig. 1).

The subjects were seated at a cephalostat and chair and their bodies were positioned according to the Frankfort horizontal plane. The subjects were instructed to perform 6 specific movements, and eyebrow movements were induced with the pupils at a midline gaze. Each action was carried out for 6 seconds and each experiment included 6 actions. The 6 movements were, in order of performance: open eyes smoothly ( 6 seconds), close eyes smoothly (6-12 seconds), gaze straight ahead (12-18 seconds), close eyes maximally (18-24 seconds), open eyes maximally (24-30 seconds), and frown maximally (30-36 seconds). All participants had the chance to practice the set of movements

\section{Fig. 1. Photogrammetric analysis}

Determining eyebrow height. A horizontal plane is drawn through the medial canthi. Vertical lines are drawn from the horizontal to the upper brow margin at the medial canthi, lateral limbus, lateral canthi, and eyebrow lateral end point. The image ruler is manipulated on the screen to measure vertical height.

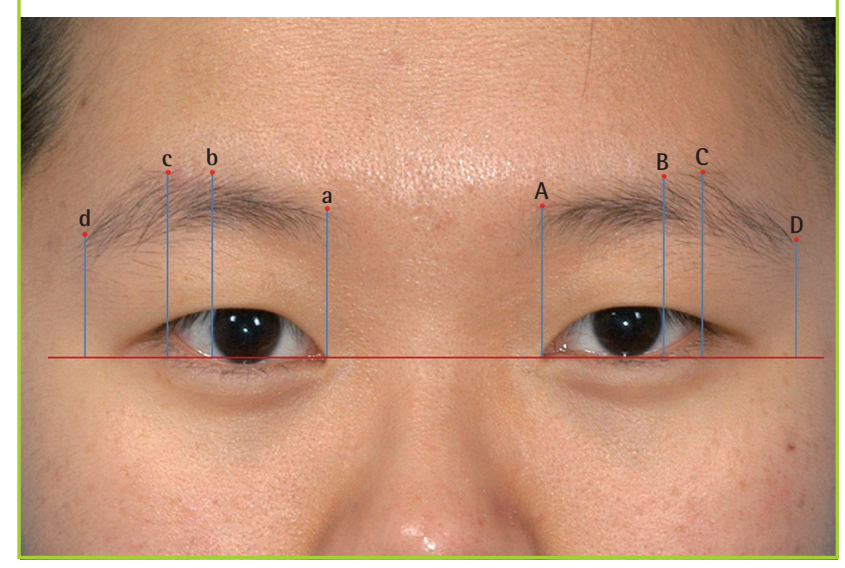

3 times, and the procedures for each action were explained in greater detail before the main experiment. Photographs were taken while the subjects adopted the series of facial poses 3 times. Videos were recorded simultaneously for later evaluation of individual motor task performance. All patients provided written consent.

\section{Photogrammetric analysis of eyebrow position}

All photographic images were analyzed with the Photoshop software package ver. 9.0 (Adobe, San Jose, CA, USA). Images were retrieved and shown on the monitor using the 'measure tool' in the 'toolbox.' The length of a 10-mm paper ruler was measured on the image, and the size of the image was adjusted using the 'image size' icon until it corresponded to $10 \mathrm{~mm}$; thus, the actual size of the image was determined [12]. Each eyebrow position was determined by measuring from a reference horizontal plane drawn between the medial canthi to 4 vertical landmarks on the upper brow margin (Fig. 1). To reduce errors, 1 investigator took measurements 3 times, and the mean of the 3 measurements was defined as the measured data.

\section{Statistical analysis}

Statistical analysis was performed using SPSS for Windows ver. 12.0 (IBM Corp, Armonk, NY, USA). An independent t-test was used for intergroup comparisons, and a paired t-test was used for intragroup comparisons. Analysis of variance was used to compare the actions. P-values $<0.05$ were considered to be statistically significant. 


\section{RESULTS}

Data from 2 of the younger and 2 of the older participants were excluded from analysis due to poor performance ability demonstrated during re-verification of the video footage. Seventy-two cases (right and left side) from 36 subjects were analyzed.

\section{Photogrammetric analysis of eyebrow position Younger group}

The positions of the left and right upper eyebrow margin were different in all experimental groups $(\mathrm{P}<0.001)$. The mean positions of the upper eyebrow at the medial canthus (MC), lateral limbus (LL), lateral canthus (LC), and lateral end point (LEP) when opening the eyes smoothly (the reference action) in the younger group ( $\mathrm{n}=36$ ) were $26.36 \mathrm{~mm}, 31.02 \mathrm{~mm}, 28.37 \mathrm{~mm}$, and $21.61 \mathrm{~mm}$, respectively. The mean positions of the upper eyebrow at the MC, LL, LC, and LEP when closing the eyes smoothly and gazing straight ahead were $26.23 \mathrm{~mm}, 30.78 \mathrm{~mm}$, $28.10 \mathrm{~mm}$, and $21.91 \mathrm{~mm}$; and $26.89 \mathrm{~mm}, 30.93 \mathrm{~mm}, 28.16$ $\mathrm{mm}$, and $21.76 \mathrm{~mm}$, respectively. These results indicate that the eyebrow was in almost the same position when the eyes were open or closed and gazing straight ahead.

The values when the eyes were opened maximally, which is the highest position, were $31.28 \mathrm{~mm}, 34.88 \mathrm{~mm}, 31.62 \mathrm{~mm}$, and $23.43 \mathrm{~mm}$, respectively. The values at maximal frown were
$23.81 \mathrm{~mm}, 26.2 \mathrm{~mm}, 25.07 \mathrm{~mm}$, and $19.89 \mathrm{~mm}$, respectively. The values when closing the eyes maximally, the lowest position, were $21.35 \mathrm{~mm}, 24.31 \mathrm{~mm}, 23.07 \mathrm{~mm}$, and $18.52 \mathrm{~mm}$, respectively. The LL was the highest position for all actions.

The maximum vertical movement of the eyebrows from the highest position (opening eyes maximally) to the lowest position (closing eyes maximally) at the MC, LL, LC, LEP was 9.93 $\mathrm{mm}, 10.57 \mathrm{~mm}, 8.55 \mathrm{~mm}$, and $4.91 \mathrm{~mm}$, respectively. The spans of movement at the MC, LL, and LC were similar. However, the change at the LEP was significantly smaller $(\mathrm{P}<0.001)$. Vertical movement from the point of the reference action (opening eyes smoothly) at the LEP, when the eyes were opened and closed maximally, was $1.82 \mathrm{~mm}$ upward and $3.09 \mathrm{~mm}$ downward $(\mathrm{P}=0.198)$ (Table 1, Fig. 2).

\section{Older group}

The mean positions of the upper eyebrow at the MC, LL, LC, and LEP when the older group opened their eyes smoothly (the reference action, $\mathrm{n}=36$ ) were $24.77 \mathrm{~mm}, 26.98 \mathrm{~mm}, 24.70 \mathrm{~mm}$, and $18.07 \mathrm{~mm}$, respectively. The values when gazing straight ahead were $25.78 \mathrm{~mm}, 28.01 \mathrm{~mm}, 25.00 \mathrm{~mm}$, and $18.06 \mathrm{~mm}$, respectively, and the $\mathrm{MC}$ and LL displacements were significant compared with the reference action point $(\mathrm{P}=0.045$ and $\mathrm{P}=$ 0.023 , respectively). That is, the medial eyebrow was in a higher position when gazing straight ahead.

Table 1. Measurements of eyebrow height in the younger and older groups at points along the upper brow margin according to the action

\begin{tabular}{|c|c|c|c|c|}
\hline Action & $\mathrm{MC}(\mathrm{mm})^{\mathrm{a})}$ & $\mathrm{LL}$ & LC & EP \\
\hline \multicolumn{5}{|l|}{ Age $20-30$} \\
\hline Open your eyes smoothly & $26.36 \pm 3.12$ & $31.02 \pm 3.06$ & $28.37 \pm 3.14$ & $21.61 \pm 3.72$ \\
\hline Close your eyes smoothly & $26.23 \pm 3.27$ & $30.78 \pm 3.47$ & $28.10 \pm 3.06$ & $21.91 \pm 3.64$ \\
\hline Gaze straight ahead & $26.89 \pm 3.42$ & $30.93 \pm 3.21$ & $28.16 \pm 3.00$ & $21.76 \pm 3.00$ \\
\hline Close your eyes maximally & $21.35 \pm 3.31$ & $24.31 \pm 3.12$ & $23.07 \pm 2.74$ & $18.52 \pm 3.27$ \\
\hline Open your eyes maximally & $31.28 \pm 4.14$ & $34.88 \pm 3.24$ & $31.62 \pm 3.61$ & $23.43 \pm 4.23$ \\
\hline Frown maximally & $23.81 \pm 2.22$ & $26.20 \pm 1.89$ & $25.07 \pm 2.12$ & $19.89 \pm 2.92$ \\
\hline \multicolumn{5}{|l|}{ Age $50-70$} \\
\hline Open your eyes smoothly & $24.77 \pm 3.26$ & $26.98 \pm 3.37$ & $24.70 \pm 2.96$ & $18.07 \pm 2.86$ \\
\hline Close your eyes smoothly & $24.12 \pm 3.00$ & $26.68 \pm 3.61$ & $24.99 \pm 3.21$ & $17.99 \pm 3.16$ \\
\hline Gaze straight ahead & $25.78 \pm 3.38$ & $28.01 \pm 3.11$ & $25.00 \pm 2.99$ & $18.06 \pm 2.86$ \\
\hline Close your eyes maximally & $21.20 \pm 2.58$ & $22.85 \pm 2.58$ & $21.64 \pm 2.72$ & $16.47 \pm 1.96$ \\
\hline Open your eyes maximally & $26.96 \pm 2.72$ & $29.55 \pm 2.82$ & $26.65 \pm 2.61$ & $18.63 \pm 2.23$ \\
\hline Frown maximally & $21.40 \pm 2.86$ & $23.01 \pm 2.70$ & $21.70 \pm 2.79$ & $16.08 \pm 2.17$ \\
\hline \multicolumn{5}{|l|}{ P-value $e^{\text {b) }}$} \\
\hline Open your eyes smoothly & 0.1895 & 0.0032 & 0.0062 & 0.0246 \\
\hline Close your eyes smoothly & 0.1476 & 0.0012 & 0.0002 & 0.0004 \\
\hline Gaze straight ahead & 0.1526 & 0.0021 & $<0.0001$ & $<0.0001$ \\
\hline Close your eyes maximally & 0.9738 & 0.1969 & 0.1867 & 0.0031 \\
\hline Open your eyes maximally & 0.0006 & $<0.0001$ & $<0.0001$ & $<0.0001$ \\
\hline Frown maximally & 0.0126 & 0.0001 & 0.0003 & $<0.0001$ \\
\hline
\end{tabular}




\section{Fig. 2. Eyebrow height according to the action}

Eyebrow height according to the action in younger $(A)$ and older (B) women. Measurements to the top of the brow margin area are shown for each point measured. No differences were observed in the younger group opening their eyes smoothly, closing their eyes smoothly, or gazing straight ahead. However the eyebrows were in a significantly higher position in the older group when they gazed straight ahead. MC, medial canthi; LL, lateral limbus; LC, lateral canthi; EP, lateral end point of eyebrow.
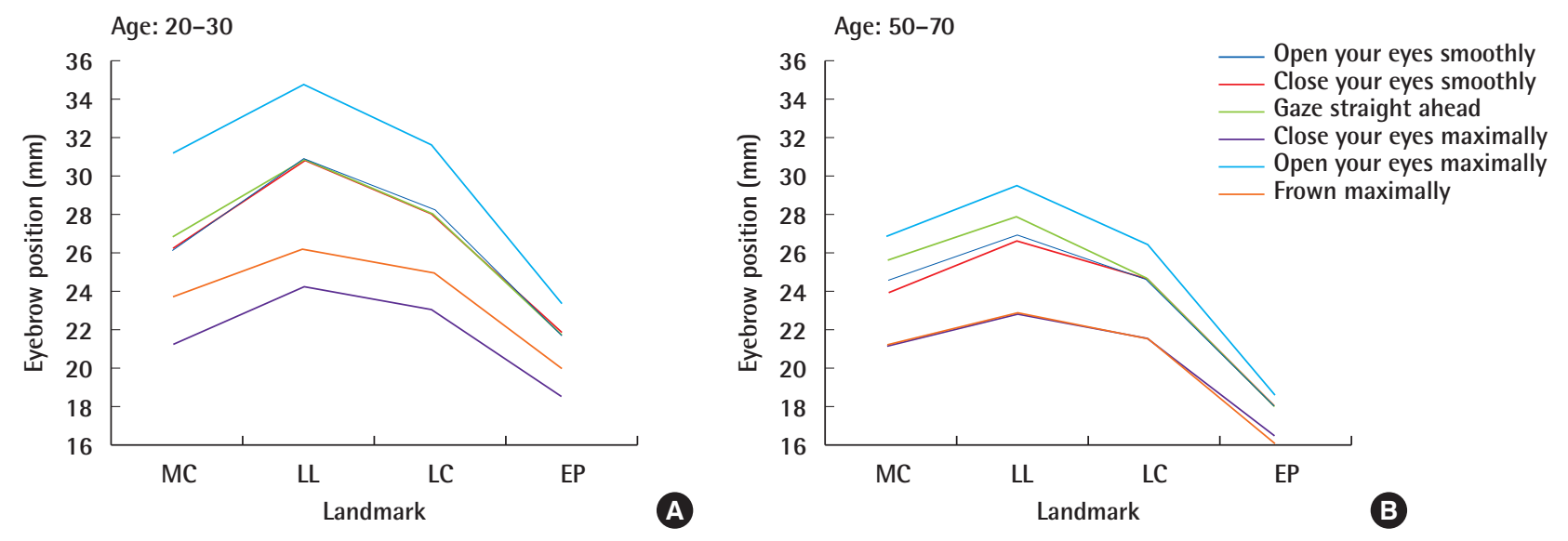

The values with eyes opened maximally, the highest position, were $26.96 \mathrm{~mm}, 29.55 \mathrm{~mm}, 26.65 \mathrm{~mm}$, and $18.63 \mathrm{~mm}$. The respective values when closing the eyes maximally and frowning maximally were $21.20 \mathrm{~mm}, 22.85 \mathrm{~mm}, 21.64 \mathrm{~mm}$, and 16.47 $\mathrm{mm}$; and $21.40 \mathrm{~mm}, 23.01 \mathrm{~mm}, 21.70 \mathrm{~mm}$, and $16.08 \mathrm{~mm}$ $(\mathrm{P}>0.05)$.

As in the younger group, the LL was in the significantly highest position for every action. The differences at the MC, LL, LC, and LEP when comparing the highest position (opening eyes maximally) with the lowest position (closing eyes maximally) were $5.76 \mathrm{~mm}, 6.7 \mathrm{~mm}, 5.01 \mathrm{~mm}$, and $2.16 \mathrm{~mm}$, respectively. As in the younger group, the spans of movement at MC, LL, and LC were similar. However, the change was significantly less at the LEP $(\mathrm{P}<0.001)$. Vertical movement at the LEP compared with the reference action (opening eyes smoothly) when the eyes were opened and closed maximally was $0.56 \mathrm{~mm}$ upward and $1.6 \mathrm{~mm}$ downward $(\mathrm{P}=0.274)$.

\section{Comparing younger and older age groups}

The eyebrows were in almost the same position when the eyes were opened and closed smoothly and gazing straight ahead in the younger group. However, the eyebrow position was the same only when the eyes were opened and closed smoothly in the older group, and the medial eyebrow was in the highest position when gazing straight ahead (Fig. 2).

The highest eyebrow position was observed when the eyes were opened maximally in both groups. However, the lowest positions of the eyebrows occurred when the eyes were closed maximally in the younger group and when the eyes were closed

\section{Fig. 3. Eyebrow height of smoothly opening eyes}

The eyebrow heights of younger and older women are shown when they opened their eyes smoothly. When eyebrow heights were compared between younger and older women, those of the older women were significantly lower, except at the $M C_{\text {, }}$ and the difference was statistically significant. MC, medial canthi; LL, lateral limbus; LC, lateral canthi; EP, lateral end point of eyebrow. ${ }^{\text {a) Statistical }}$ significance.

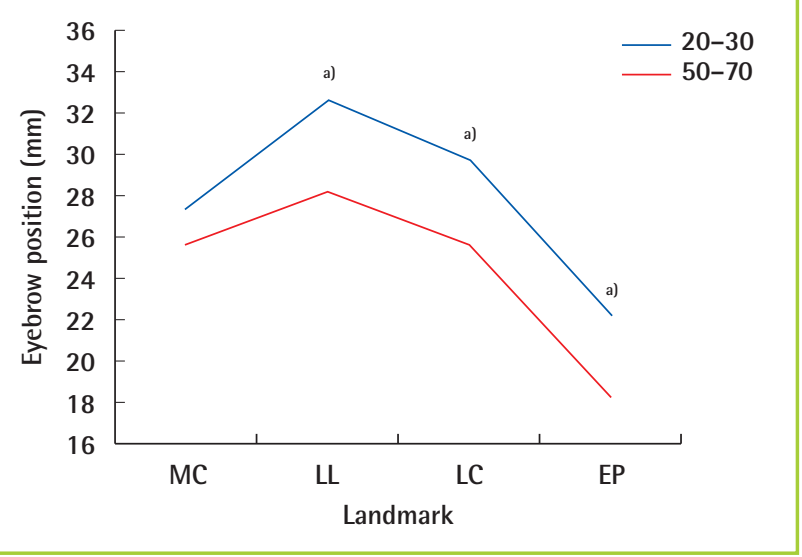

maximally and frowning maximally in the older group.

When participants opened their eyes smoothly, the differences in eyebrow height at MC, LL, LC, and LEP were $1.59 \mathrm{~mm}, 4.04$ $\mathrm{mm}, 3.67 \mathrm{~mm}$, and $3.54 \mathrm{~mm}$, respectively. When the eyes were closing smoothly and gazing straight ahead, the values were 2.11 $\mathrm{mm}, 4.1 \mathrm{~mm}, 3.11 \mathrm{~mm}$, and $3.92 \mathrm{~mm}$; and $1.11 \mathrm{~mm}, 2.92 \mathrm{~mm}$, $3.16 \mathrm{~mm}$, and $3.7 \mathrm{~mm}$, respectively. No differences were observed among these 3 actions at the $\mathrm{MC}(\mathrm{P}=0.19, \mathrm{P}=0.148$, and $\mathrm{P}=0.153)$. However, the older group showed significantly lower brow positions at the LL, LC, and LEP (Figs. 3-5). 


\section{Fig. 4. Eyebrow height of smoothly closing eyes}

The eyebrow heights of younger and older women are shown when they closed their eyes smoothly. The eyebrow heights of younger women were significantly higher than those of older women, except at the MC. MC, medial canthi; LL, lateral limbus; LC, lateral canthi; EP, lateral end point of eyebrow. ${ }^{\text {a) }}$ Statistical significance.

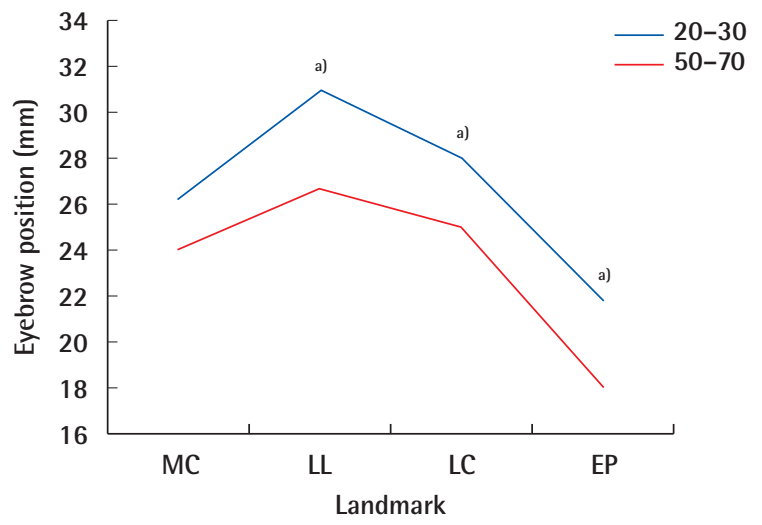

\section{Fig. 5. Eyebrow height of eyes gazing straight}

The eyebrow heights when subjects gazed straight ahead. Eyebrow heights were significantly lower in older women than in younger women, except at the MC. MC, medial canthi; LL, lateral limbus; LC,

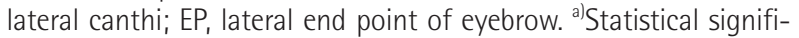
cance.

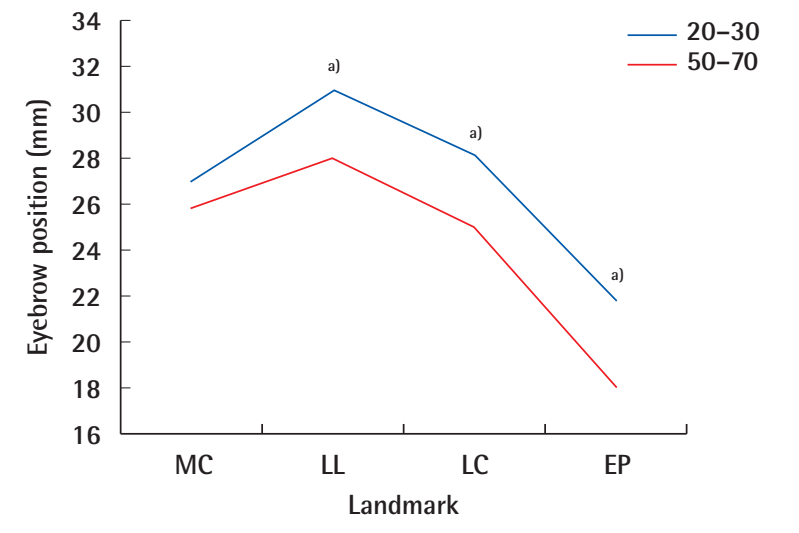

The differences when eyes were closing maximally at the $\mathrm{MC}$, LL, LC, and LEP were $0.15 \mathrm{~mm}, 1.46 \mathrm{~mm}, 1.43 \mathrm{~mm}$, and 2.05 $\mathrm{mm}$ respectively, indicating that only the lateral $\mathrm{EP}$ had lower brow positions in the older group ( $\mathrm{P}=0.003)$ (Fig. 6).

The differences in the older group when the eyes were opened maximally and frowning maximally were $4.32 \mathrm{~mm}, 5.33 \mathrm{~mm}$, $4.97 \mathrm{~mm}$, and $4.8 \mathrm{~mm}$; and $2.41 \mathrm{~mm}, 3.19 \mathrm{~mm}, 3.37 \mathrm{~mm}$, and $3.81 \mathrm{~mm}$, respectively, indicating that every point measured had a lower position. Generally, the eyebrows were lower in the older group for the same action (Figs. 7, 8).

The lengths of vertical movement of the eyebrows at the MC, LL, LC, and LEP were also smaller in the older group at 5.76

\section{Fig. 6. Eyebrow height with eyes closed maximally}

Only the height of the EP in older women was significantly lower than that of younger women. MC, medial canthi; LL, lateral limbus; $\mathrm{LC}$, lateral canthi; EP, lateral end point of eyebrow. ${ }^{\text {a) }}$ Statistical significance.

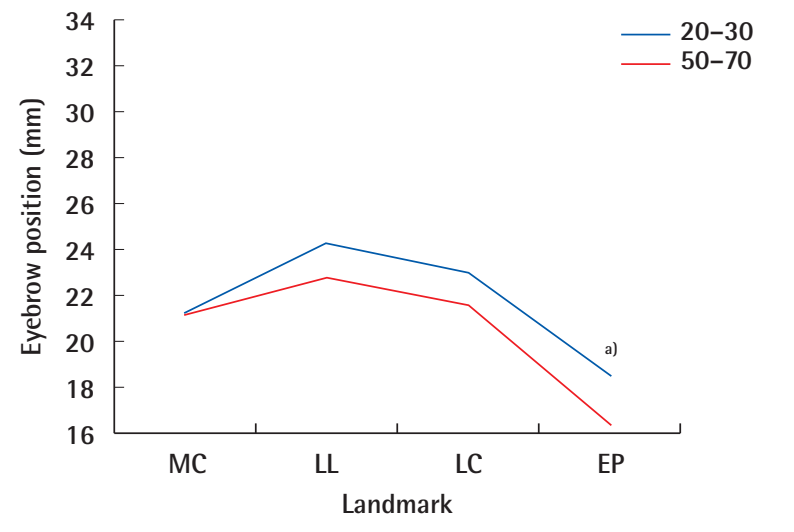

\section{Fig. 7. Eyebrow height with eyes opened maximally}

Eyebrow heights of older women were significantly lower than those of younger women for every point measured. MC, medial canthi; $L L$, lateral limbus; $L C$, lateral canthi; $E P$, lateral end point of eyebrow. ${ }^{\text {a) }}$ Statistical significance.

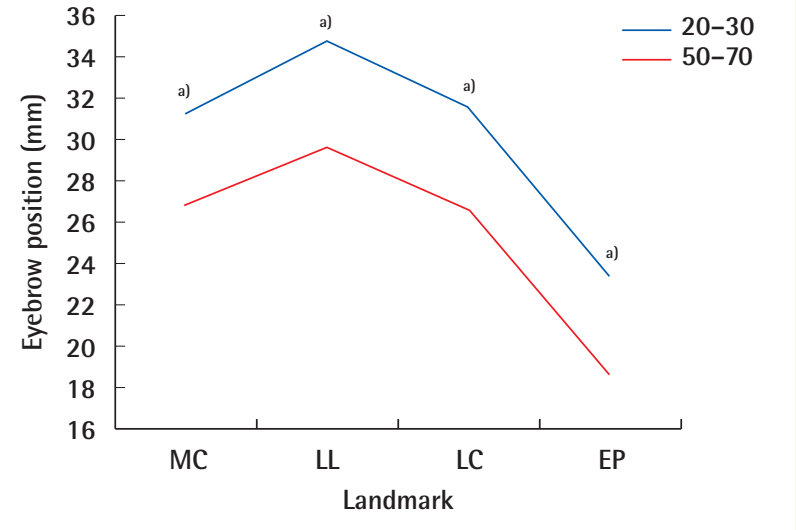

$\mathrm{mm}, 6.7 \mathrm{~mm}, 5.01 \mathrm{~mm}$, and $2.55 \mathrm{~mm}$, respectively, whereas values for the younger group were $9.93 \mathrm{~mm}, 10.57 \mathrm{~mm}, 8.55 \mathrm{~mm}$, and $4.91 \mathrm{~mm}$, respectively $(\mathrm{P}<0.001$ for $\mathrm{MC}, \mathrm{P}<0.001$ for $\mathrm{LL}$, $\mathrm{P}<0.001$ for $\mathrm{LC}, \mathrm{P}<0.001$ for LEP).

\section{DISCUSSION}

Facial skin aging occurs for 2 reasons. First, the skin and ligamentous structures, which have lost elasticity, sag downward under the influence of gravity. Second, endogenic changes occur, which are caused by the atrophy of elastic fibers and fat loss, abnormal pigmentation, thinning of the scarfskin, and changes due to ultraviolet radiation $[13,14]$. A uniform descent of the 


\section{Fig. 8. Eyebrow height when frowning maximally}

Eyebrow heights at all points in older women were significantly lower than those in younger women. MC, medial canthi; LL, lateral

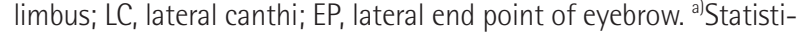
cal significance.

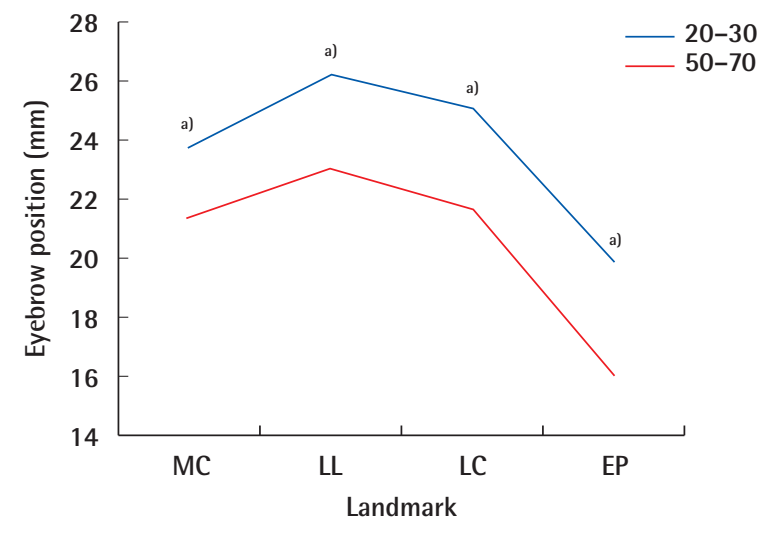

eyebrow is observed with aging as a result of these 2 factors, even in the periorbital areas. However, whether there is a change in eyebrow position, and what kind of change occurs, is controversial. Van den Bosch et al. [15] reported that eyebrow height over the pupil increased with aging in a cross-sectional cohort study of 320 men and women aged 10-89 years. On the other hand, Goldstein and Katowitz [16] found that eyebrow height over the pupil and lateral canthus remains in a similar position over time. Price et al. [17] showed that eyebrow height over the LL remains in a similar position over time. Lambros [18] evaluated the longitudinal effects of aging at individual eyebrows by overlaying photographs and subjectively examined them using digital animation to avoid possible measurement errors. Twenty-eight percent of the subjects showed an elevated eyebrow position, $41 \%$ remained stable, and $29 \%$ showed a descent.

There are several possible reasons for the varied results in the measurement of the effect of eyebrow position on aging. It is conceivable that the positions were not measured under the same conditions. As eyebrows are not static, but dynamic, their shape can vary according to experimental conditions and the emotional state of the participants [19]. Therefore, measurements must be taken for the same actions, which can be challenging in practice. Even if an experiment is performed in the same position, habitual actions, such as frowning or raising an eyebrow, often occur [8]. Our experiment was prospectively performed to obtain objective results; participants were sufficiently trained before the experiment, and the 6 actions of the eyebrow were measured in detail. Video footage was re-verified. If a participant had incorrectly performed the action for the proper duration, the participant was excluded from the experi- ment. As overall changes in the eyebrows cannot be identified by simply measuring 1 part, 4 landmarks were measured for analysis in our experiment.

Our results showed no difference in the height of the eyebrows at the MC when opening and closing the eyes smoothly, gazing straight ahead, and closing the eyes maximally, indicating that the MC maintains its position anatomically, and the muscles related to the MC maintain their balance well. However, the eyebrow position of the MC was significantly lower when the eyes were opened maximally and frowning was maximal, indicating that the corrugator supercilii muscle at the $\mathrm{MC}$ had lower action potential.

In contrast, eyebrow height was significantly lower at the lateral end point during all actions. The causes for this may include skin condition and the interdigitated relationships between the frontalis muscle, OOM, and CSM inserted into the dermis. Those muscles have reverse actions that maintain the normal balanced position of the eyebrow for dynamic equilibrium, akin to muscles playing 'tug-of-war' due to their interdigitated anatomy. However, these states of dynamic equilibrium are not achieved in the lateral segment, because of the lack of insertion of the frontalis muscle; this leads to an earlier appearance of sagging in the eyebrows. Knize [9] reported that lateral ptosis occurs when eyebrow height is maintained where the frontalis is suspended. Ptosis occurs faster in the lateral segment because of the absence of muscle suspension in the area. However, the function of the frontalis muscle is not simple static suspension.

Yun et al. [20] reported that strained expressions of the eyebrow impose increased motor recruitment in the frontalis, $\mathrm{OOM}$, and CSM. Motor recruitment to the frontalis and corrugator supercilii muscles decreases with advancing age because of age-related muscular weakness. However, orbicularis oculi muscle motor recruitment is preserved or even increases. Our results also show that there was less vertical movement of the eyebrows in the older group than that in the younger group. The weakness of muscles related with eyebrow position may be attributed to a decrease in the length of vertical movement of the eyebrow with aging. However, no difference was observed between the younger and older groups, except with the EP, when the eyes were closed maximally, because orbicularis oculi muscle function is preserved with aging.

This study examined eyebrow positions and vertical movement potential. For eyebrow position, the position of the lateral EP of the older group was significantly lower than that of the younger group during all 6 actions. However, no difference in eyebrow position between the younger and older group was observed at the MC during smooth opening and closing of the eyes, forward gaze, or maximal closing of the eyes. And no dif- 
ference was observed when the eyes were closed maximally at 3 landmarks, with the exception of the EP. The vertical movement of the eyebrows at the 4 landmarks decreased with aging. And the least vertical movement was observed at the lateral EP in both age groups.

In this study, the changes with aging in eyebrow position at the lateral EP were greater than at the MC. The height of the lateral EP decreased with aging. However, the least vertical movement in the lateral EP was found in the same group. The lateral EP was the most static structure of the 4 landmarks. Therefore, it is important to focus on correction of the lateral EP for periorbital rejuvenation.

\section{REFERENCES}

1. Ellis DA, Masri H. The effect of facial animation on the aging upper half of the face. Arch Otolaryngol Head Neck Surg 1989;115:710-3.

2. Westmore M. Facial cosmetics in conjunction with surgery. Proceedings of the Aesthetic Plastic Surgical Society Meeting; May; 1974; Vancouver, British Columbia.

3. Ellenbogen R. Transcoronal eyebrow lift with concomitant upper blepharoplasty. Plast Reconstr Surg 1983;71:490-9.

4. Brennan HG. Correction of the ptotic brow. Otolaryngol Clin North Am 1980;13:265-73.

5. Tolleth H. Concepts for the plastic surgeon from art and sculpture. Clin Plast Surg 1987;14:585-98.

6. Angres GG. Blepharopigmentation and eyebrow enhancement techniques for maximum cosmetic results. Ann Ophthalmol 1985;17:605-11.

7. Baker SB, Dayan JH, Crane A, et al. The influence of brow shape on the perception of facial form and brow aesthetics. Plast Reconstr Surg 2007;119:2240-7.

8. Lambros V. Discussion: changes in eyebrow position and shape with aging. Plast Reconstr Surg 2009; 124:1302-3.

9. Knize DM. An anatomically based study of the mechanism of eyebrow ptosis. Plast Reconstr Surg 1996;97:1321-33.

10. Hop JW, Frijns CJ, van Gijn J. Psychogenic pseudoptosis. J Neurol 1997;244:623-4.

11. Knize DM. Anatomic concepts for brow lift procedures. Plast Reconstr Surg 2009; 124:2118-26.

12. Han K, Kwon HJ, Choi TH, et al. Comparison of anthropometry with photogrammetry based on a standardized clinical photographic technique using a cephalostat and chair. J Craniomaxillofac Surg 2010;38:96-107.

13. Uitto J. Connective tissue biochemistry of the aging dermis: age-related alterations in collagen and elastin. Dermatol Clin 1986;4:433-46.

14. Fenske NA, Lober CW. Structural and functional changes of normal aging skin. J Am Acad Dermatol 1986;15:571-85.

15. Van den Bosch WA, Leenderd I, Mulder P. Topographic anatomy of the eyelids, and the effects of sex and age. $\mathrm{Br}$ Ophthalmol 1999;83:347-52.

16. Goldstein SM, Katowitz JA. The male eyebrow: a topographic anatomic analysis. Ophthal Plast Reconstr Surg 2005;21: 285-91.

17. Price KM, Gupta PK, Woodward JA, et al. Eyebrow and eyelid dimensions: an anthropometric analysis of African Americans and Caucasians. Plast Reconstr Surg 2009;124:615-23.

18. Lambros V. Observations on periorbital and midface aging. Plast Reconstr Surg 2007;120:1367-76.

19. Schwartz GE, Fair PL, Mandel MR, et al. Facial electromyography in the assessment of improvement in depression. Psychosom Med 1978;40:355-60.

20. Yun S, Son D, Yeo H, et al. Changes of eyebrow muscle activity with aging: functional analysis revealed by electromyography. Plast Reconstr Surg 2014;133:455e-463e. 